\title{
Acrocianosis como forma de debut de una esclerosis sistémica progresiva
}

\author{
M. FEITO RODRÍGUEZ, J. GARCÍA MACARRÓN, M. A. MARTÍN DÍAZ, C. RUBIO \\ FLORES, C. VIDAURRÁZAGA DÍAZ-ARCAYA
}

Servicio de Dermatología. Hospital Universitario La Paz. Madrid

\author{
ACROCYANOSIS AS A FORM OF PRESENTATION OF SYSTEMIC \\ SCLEROSIS
}

\section{RESUMEN}

La acrocianosis es un cuadro clínico caracterizado por una coloración azul violácea persistente y simétrica de zonas acras, principalmente manos y pies, que no está precedida por episodios de palidez previos. Es una entidad que puede asociarse a diversas causas subyacentes, pero es infrecuente que lo haga como forma de debút de una esclerosis sistémica progresiva.

Se presenta a continuación un nuevo caso de esta patología y se comentan sus características etiopatogénicas, clínicas y diagnósticas más relevantes además de un intento de diferenciar este término de otros con los que existe confusión en la literatura.

PALABRAS CLAVE: Acrocianosis. Esclerosis sistémica. Esclerodermia.

\section{ABSTRACT}

Acrocyanosis is an infrequent entity characterized by persistent and symmetrical erythrocyanotic discoloration of the hands, feet and face, which is not preceded by episodes of previous paleness. Acrocyanosis can be secondary to a variety of underlying causes, but is uncommon as a form of presentation of systemic sclerosis.

We present a new clinical case of this disease and discuss its etiopathogenic, clinical, diagnostic and therapeutic characteristics; in addition to, we seek to distinguish this term from others that might be confused in the literature.

KEY WORDS: Acrocyanosis. Systemic sclerosis. Scleroderma.

Feito Rodríguez M, García Macarrón J, Martín Díaz MA, Rubio Flores C, Vidaurrázaga Díaz-Arcaya C. Acrocianosis como forma de debut de una esclerosis sistémica progresiva. An Med Interna (Madrid) 2006; 23: 490-492.

\section{INTRODUCCIÓN}

La acrocianosis se define como la coloración persistente eritrocianótica de manos y pies, frecuentemente con un patrón moteado (1). Los cambios suelen ser transitorios tras exposición al frío, pero frecuentemente es persistente durante el invierno e incluso durante la primavera y el verano. La acrocianosis se presenta en un número importante de casos asociado a diversas causas subyacentes, entre ellas la esclerosis sistémica progresiva.

Presentamos a continuación el caso de un varón de 39 años, que consultó por coloración violácea simétrica y persistente en extremos distales de dedos de ambas manos de 15 días de evolución, asociado a frialdad de las mismas, sin otra sintomatología de interés.

\section{CASO APORTADO}

Acude a nuestra consulta un varón de 39 años, sin antecedentes personales de interés salvo hipotiroidismo subclínico, remitido desde
Atención Primaria para valoración de coloración violácea en falange distal del segundo, tercer y cuarto dedo de ambas manos de 15 días de evolución, además de sensación de frialdad en las zonas referidas; las lesiones eran persistentes tanto en ambientes cálidos como en ambientes fríos, y no se objetivaban episodios de palidez previa ni rubor. No se encontraron antecedentes familiares de interés. El paciente no estaba tomando ninguna medicación vasoconstrictora.

A la exploración física se objetivó coloración violácea y frialdad en extremos distales de ambas manos de forma bastante simétrica, siendo dichos cambios más notorios en segundo, tercer y cuarto dedo (Figs. 1 y 2). Los pulsos distales se hallaban conservados, y no existían otras zonas anatómicas afectas. En este momento no se objetivó esclerodactilia, cicatrices puntiformes ni otros hallazgos significativos que sugirieran esclerodermia. La exploración cardiopulmonar no reveló ninguna anomalía.

Se realizaron varias pruebas complementarias, analíticas y de imagen. Hemograma, electrolitos, coagulación, función hepática, función renal, gasometría arterial, análisis de orina, velocidad de sedimentación globular, niveles de complemento, inmunoglobulinas, crioaglutininas, crioglobulinas y marcadores tumorales fueron normales. Un estudio de función tiroidea objetivó hipotiroidismo subclí- 


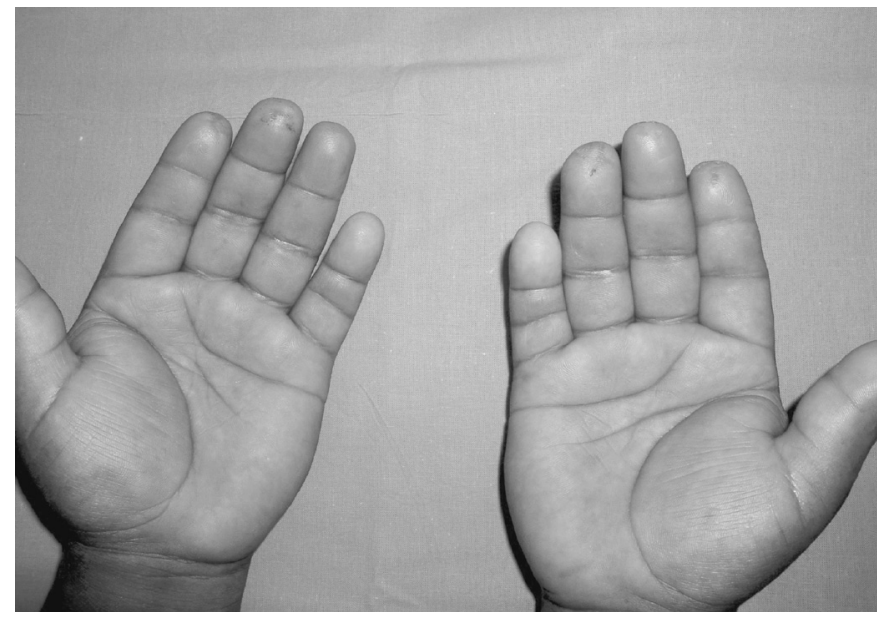

Fig. 1. Foto pretratamiento: obsérvese la coloración cianótica de pulpejos de segundo, tercer y cuarto dedo de ambas manos.

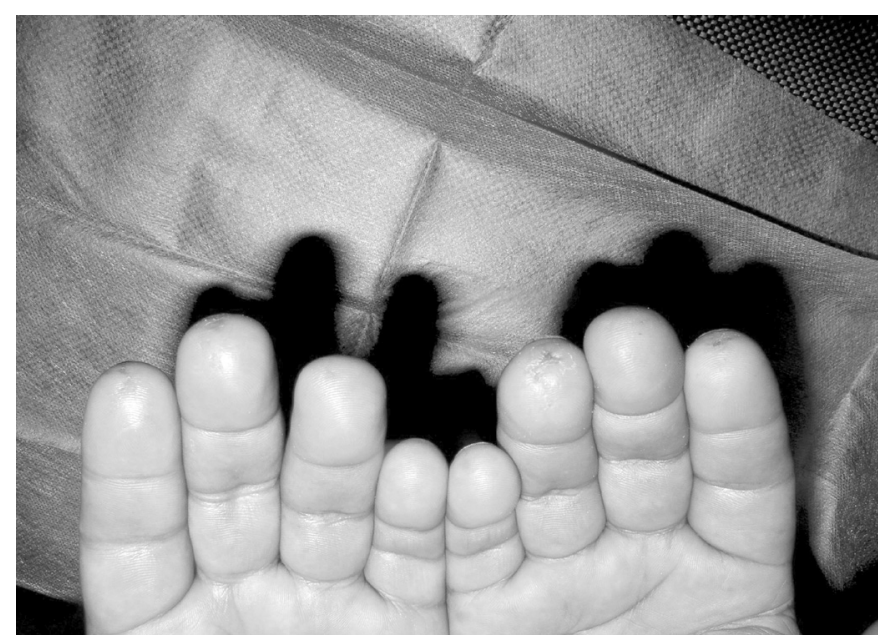

Fig. 2. Foto tras tratamiento: desaparición de la coloración cianótica. Se visualizan cicatrices puntiformes y discreta esclerodactilia.

nico, con anticuerpos antitiroglobulina y antiperoxidasa normales. Las serologías de VHB, VHC, VIH y sífilis resultaron negativas.

El estudio inmunológico reveló ANA positivos a título 1/2560 con patrón nucleolar y anticuerpos antiDNA, ENA, anticardiolipina, sc170, anticentrómero y factor reumatoide negativos. El TC torácico fue rigurosamente normal.

Dos meses después el paciente comenzó a presentar esclerodactilia y cicatrices puntiformes en pulpejos de dedos e induración de piel preesternal y región laterocervical de forma simétrica. Se inició tratamiento con pentoxifilina, corticoides orales en pauta descendente y azatioprina, con buen control de su patología.

\section{DISCUSIÓN}

En primer lugar debemos diferenciar la acrocianosis de otras entidades como la cianosis periférica, la enfermedad vascular periférica y el fenómeno de Raynaud, algo que en muchas ocasiones resulta confuso en la literatura. La cianosis periférica, secundaria a hipoxemia, se caracteriza por una gasometría arterial y/o estudio cardiopulmonar anormal; unos pulsos distales disminuidos y/o una exploración vascular alterada (2) encaminarían el diagnóstico hacia una enfermedad vascular periférica. Por último, el fenómeno de Raynaud se define como episodios de isquemia digital en respuesta al frío o a ciertos estímulos emocionales, y se caracteriza clínicamente por episodios de palidez seguidos secuencialmente de cianosis y rubor $(2,3)$.

En segundo lugar, antes de llegar al diagnóstico de acrocianosis idiopática deben descartarse diversos procesos expuestos en la tabla I (2,4-9). Hacemos mención especial a los casos de acrocianosis asociados a malignidad subyacente; éstos suelen presentarse en personas de mediana edad con factores de riesgo reconocibles, siendo los tipos más frecuentes el adenocarcinoma de pulmón y ovario. En nuestro caso fueron descartadas todas las posibles causas incluídas posibles neoplasias que pueden coincidir o aparecer posteriormente (10).

\section{TABLA I}

\section{ENTIDADES SUBYACENTES ASOCIADAS A ACROCIANOSIS}

\begin{tabular}{ll}
\hline Alteraciones vasculares & Conectivopatías \\
Infecciones (sífilis) & $\begin{array}{c}\text { Trastornos psiquiátricos (anorexia } \\
\text { nerviosa) }\end{array}$ \\
Neoplasias & Déficit metabólico \\
Medicación (imipramina) & Anticuerpos antifosfolípido \\
Crioglobulinemia, crioaglutinemia & Idiopático \\
\hline
\end{tabular}

El factor fisiopatológico más relevante en la acrocianosis es una disminución del flujo sanguíneo acral en la que parecen estar implicados diversos factores como ciertas anormalidades en la viscosidad sanguínea, defectos arteriolares y/o venosos e incluso recientemente se han implicado niveles elevados de endotelina o respuestas inapropiadas de la misma al frío como causantes de esta patología $(1,2)$. De hecho, cuando se efectúan capilaroscopias en pacientes con acrocianosis, los fenómenos que se objetivan consisten en hemorragias, edema pericapilar y capilares anormalmente dilatados (11).

Con este estudio se estableció la posibilidad de acrocianosis como forma de debút de una esclerosis sistémica progresiva sin cumplimiento de criterios en el momento del diagnóstico. La acrocianosis es una forma infrecuente de presentación de la esclerosis sistémica progresiva; sin embargo, en casi un 90\% de los pacientes encontramos presente el fenómeno de Raynaud, generalmente precediendo su comienzo, siendo la ausencia del mismo un factor de mal pronóstico de la enfermedad $(3,12)$. La detección de ciertos autoanticuerpos puede ser muy útil en el diagnóstico; al igual que en nuestro caso, la mayoría de pacientes tienen títulos elevados de anticuerpos antinucleares (ANA), resultando el patrón antinucleolar el más específico. Otros anticuerpos que pueden resultar elevados son la topoisomerasa ( $\mathrm{scl} 70)$, anticentrómero o ARN polimerasa I y III. Los criterios que se usan para el diagnóstico de ESP son los criterios de la Academia Americana de Reumatología (13); la esclerodermia proximal a las articulaciones metacarpofalángicas es el criterio mayor y existen tres crite- 
rios menores: esclerodactilia, fibrosis pulmonar bibasal y cicatrices puntiformes en los pulpejos de los dedos. Al inicio, nuestro paciente no cumplía ningún criterio, presentándose sólo con acrocianosis; rápidamente desarrolló esclerodactilia y cicatrices puntiformes junto a áreas de induración simétrica y brillante en región preesternal y laterocervical, que hacían claro el diagnóstico.

El tratamiento del cuadro acral descrito en la literatura y de

\section{Bibliografía}

1. Dowd, PM. Reactions to cold. In: Burns T, Breathnach S, Cox N, Griffiths C, editors. Rook's Textbook of Dermatology. 6nd ed. Oxford: Blackwell Publising; 2004. p. 962-971.

2. Nousari HC, Kimyai-Asadi A, Anhalt GJ. Chronic idiopathic acrocyanosis. J Am Acad Dermatol 2001; 45: S207-8.

3. Carpentier PH. Definition and epidemiology of vascular acrosyndromes. Rev Prat 1998; 48: 1641-6.

4. Gibbs MB, English III JC, Zirwas MJ. Livedo reticularis: An update. J Am Acad Dermatol 2005; 52: 1009-19.

5. Lotti T, Ghersetich I, Comacchi C, Jorizzo JL. Cutaneous small-vessel vasculitis. J Am Acad Dermatol 1998; 39: 667-87.

6. Bhanji S, Mattingly D. Acrocyanosis in anorexia nerviosa. Postgrad Med J 1991; 67: 33-5.

7. Garcia-Silva MT, Campos Y, Ribes A, Briones P, Cabello A, Santos Borbujo J, et al. Encephalopathy, petechiae, and acrocyanosis with ethylmalonic aciduria associated with muscle cytochrome c oxidase deficiency. J Pediatr 1994; 125: 843-4. acuerdo con la fisiopatología de la enfermedad consiste en antagonistas del calcio, nifedipino y pentoxifilina. Por otro lado, en el tratamiento de la esclerosis cutánea se han usado diversos agentes, algunos de ellos de dudosa eficacia, tales como penicilamina, interferón, relaxina, corticoides, metotrexate, azatioprina, ciclosporina o clorambucil. En el momento actual, nuestro paciente se encuentra controlado con pentoxifilina, corticoides orales en pauta descendente y azatioprina.

8. Anderson RP, Morris BA. Acrocyanosis due to imipramine. Arch Dis Child 1988; 63: 204-5.

9. Federman DG, Valdivia M, Kirsner RS. Syphilis presenting as the "blue toe syndrome". Arch Intern Med 1994; 154: 1029-31.

10. Poszepczynska-Guigné E, Viguier M, Chosidow O, Orcel B, Emmerich J, Dubertret L. Paraneoplastic acral vascular syndrome: Epidemiologic features, clinical manifestations, and disease sequalae. J Am Acad Dermatol 2002; 47: 47-52.

11. Monticone G, Colonna L, Palermi G, Bono R, Puddu P. Quantitative nailfold capillary microscopy findings in patients with acrocyanosis compared with patients having systemic sclerosis and control subjects. J Am Acad Dermatol 2000; 42: 787-790.

12. Falanga, V. Esclerosis sistémica. In: Bolognia JL, Jorizzo JL, Rapini RP, editors. Dermatología. 1nd ed. Londres: Mosby; 2003. p. 625-632.

13. Medsger TA Jr. Systemic sclerosis. In: Koopman WH, ed. Arthritis and Allied Conditions. Baltimore, MD: Williams and Wilkins; 1997: 143355. 